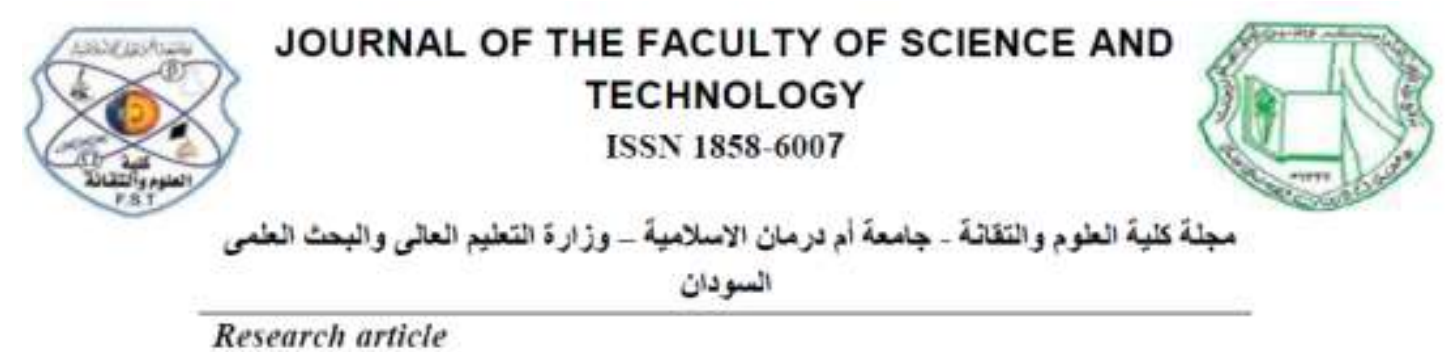

\title{
A Computer Simulation of Lithium Doped in nanosheet zinc oxide
}

\author{
Abdalla Abdelrahman Mohamed ${ }^{1}$, Bedor Mohammed Khairalla ${ }^{2}$ \\ ${ }^{1}$ E-mail: abdallam194@gmail.com \\ ${ }^{2}$ E-mail: Bedonano2019@gmqil.com
}

\begin{abstract}
The capabilities of computer through the methodologies of scientific computing used to solve many manufacturing difficulties of semiconductor materials, across all disciplines, because it is low cost and the availability of resources. In this paper, investigation of unique electronic property of zinc oxide sheet, which can be an attractive semiconductor material for many electronic devices applications, is carried out. The electronic structure of zinc oxide surface and the effect of substituting lithium atoms using CRYSTAL06 code showed that the bulk $\mathrm{ZnO}$ band gap $\approx 3.3 \mathrm{eV}$ which is in good agreement with experimental results $\approx 3.4 \mathrm{eV}$. After generating slab structure, the band gap has been increased to $5.5 \mathrm{eV}$, exhibiting high resistivity surface. Substituting of lithium to slab has decreased band gap to $4.4 \mathrm{eV}$. The results shows that there is reduction in band-gap and decreases in resistivity, predicting that Small amount of Lithium could change the zinc oxide from wide band semiconductor to half-metal compound.
\end{abstract}

Keywords: Simulation; Lithium; Doping; Nanosheet; wurtzite Zinc oxide.

\subsection{Introduction}

Zinc oxide $(\mathrm{ZnO})$ is an inorganic compound, is a white powder that is insoluble in water[(Coleman and Jagadish, 2006), in a form of a wurtzite structure with space group P63 mc in which both $\mathrm{Zn}$ and $\mathrm{O}$ sub lattices have the hexagonal close-packed structure. Its use as a widely diverse functional material, is enhanced by the fact that it can be grown in bulk, thin film, and nanostructures, examples of the latter being nanowires, nanobelts, and other morphologies, and products including rubbers, plastics, ceramics, glass, cement, lubricants, paints, ointments, adhesives, sealants, pigments, foods, batteries, ferrites, fire retardants, and first-aid tapes("Physical Electronics and Nanotechnology - L Linköping University," n.d.). As the advancement in nanotechnology continues, the use of $\mathrm{ZnO}$ nanoparticles in sunscreen products has proven to be safer (with lower toxicity) than conventional UV-blocking sunscreens when applied to the skin[(Moezzi et al., 2012)]. Experimentally, when Lithium ion replaced $\mathrm{Zn}$ ion in wurtzite $\mathrm{ZnO}$, it causes the dielectric anomaly and decreases the A.C conductivity, increase the resistivity of $\mathrm{ZnO}$ and leakage current densities, indicating that the ferroelectricity was caused by the $\mathrm{Li}$ concentration[ (Kim et al., 2011).Lithium may behave both as a 
donor and acceptor in $\mathrm{ZnO}$, when substituting for $\mathrm{Zn}$ acting as acceptor[ ("Wall-E, AJa HGVd, Fundamental of Zinc Oxide as Semiconductor. 2009. بحث Google," n.d.)]. Several groups have reported first-principles calculations for column-IA impurities ( $\mathrm{Li}, \mathrm{Na}$ and $\mathrm{K}$ ) in $\mathrm{ZnO}$ Park et al performed calculations for substitutional and interstitial $\mathrm{Li}, \mathrm{Na}$, and $\mathrm{K}$ in $\mathrm{ZnO}$, and reported ionization energies of 0.09 eV for substitutional Li[("Phys. Rev. B 66, 073202 (2002) - Origin of p-type doping difficulty in $\mathrm{ZnO}$ : The impurity perspective," n.d.), ("Phys. Rev. B 71, 155205 (2005) - Theory of Li in ZnO: A limitation for Li-based \$p\$-type doping," n.d.)]. Computer Simulation packages, based on ab-initio calculation method with Hartree-Fock or KohnSham Hamiltonians (that adopt an Exchange - Correlation functional PBE following the postulates of DensityFunctional theory and B3LYP [(“crystal17.pdf," n.d.)] hybrid functional is used to study the structural and electrical properties of doping lithium ion in $\mathrm{ZnO}$ surface. This contribute to minimize the cost of material focused on Zinc oxide semiconductors in forms a wurtzite structure, and to obtain new properties of $\mathrm{ZnO}$ nanosheet to manufacture new structures with more conductivity, and advance semiconductor devices.

\section{Methodology}

CRYSTAL06 [R. Dovesi, V.R.S., C.Roetti, R.Orlando,] employs atomcentered Gaussian-type function as basis sets. The basis sets used were allelectron sets on $\mathrm{Zn}(86-411 \mathrm{~d} 41)$, O (8411-d1) and a pseudopotential basis set (HAYWSC-31(3d)) was used for Li to reduce computational effort to calculate $\mathrm{ZnO}$ as a crystal in periodic system in space group (p $63 \mathrm{~m} \mathrm{c}$ ) and lattice parameters (angstroms and degrees) conventional cell $\mathrm{A}=\mathrm{B}=3.24931$ and $\mathrm{C}=5.20571$ and expansion matrix of primitive cell $2 \times 2 \times 5$ to generate 80 atoms per supercell of $\mathrm{ZnO}$, lattice parameters (angstrom and degrees) $\mathrm{A}=\mathrm{B}=6.49862$ and $\mathrm{C}=$ 26.02854 Figure 4.1 below displays supercell structure of $\mathrm{ZnO}$.

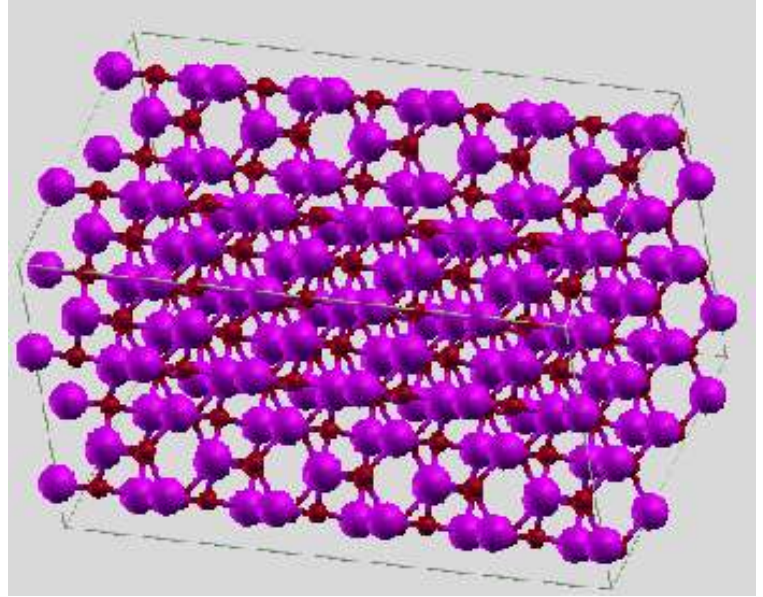

Figure1: 2x2x5 Bulk ZnO supercell crystal structure

generate slab from crystal using SLABCUT option, surface (l $\left.\begin{array}{lll}1 & 0\end{array}\right), 2$ layer, crystal calculation generated super cell two dimensional slab parallel to the selected plane, slab generated and SCF ended and converged on energy -
$3.7090200183929 \mathrm{e}+04 \quad$ cycles 27, calculation take 5 days.

$\mathrm{XCrysden}$ is a program to visualize crystal structures, iso-surface of 3D data and molecular, aiming at display of isosurfaces and contours, which can be superimposed on crystalline structures 
and interactively rotated and manipulated, the name of the program stands for Crystalline Structures and Densities and $\mathrm{X}$ because it runs under the X-Window environment. it facilitates a display of isosurfaces and contours, which can be superimposed on crystalline structures and interactively rotated and manipulated. It also possesses some tools for analysis of properties in reciprocal space such as interactive selection of $\mathrm{k}$-paths in the Brillouin zone for the band-structure plots, and visualization of Fermi surfaces, XcrySDen is able to: switch between primitive and conventional cell settings, change the number of the displayed unit cells, that is, display smaller or larger portions of a crystal, display the crystal lattice, visualize the Wigner-Seitz cell and Brillouin-zone and more [("XCrySDen- (X-Window) Crystalline Structures and Densities," n.d.).

\section{Results and Discussion}

\subsection{Density of state band structures of}

\section{Bulk ZnO}

Band structure is electronic band structure and energy schema to describe the conductivity of conductor, insulator and semiconductors.

The schema consists of three energy band (conduction-band and valenceband) and forbidden energy band, the valence electron which serve as charge carriers are located in valence band, in the ground state the conduction band is occupied with no electron, between two energy band there is band-gap its width effects the conductivity of materials. This is produced by solving the KS equations, for a collection of $\mathrm{k}$-vector points that follow a path along the border of theBZ1.

The band gap of bulk $\mathrm{ZnO}$ is calculated by using high symmetry G-M-K-G-A-LH-A-L-M-K-Has shown in figure2. According to the calculation, the band gap of bulk $\mathrm{ZnO}$ is finding to be direct band gap $\approx 3.3 \mathrm{eV}$, Figure 3 show the total density of state which is support the results of band structure calculation, and noted the $\mathrm{Zn} 3 \mathrm{~d}$ is predominant state near Fermi energy level.

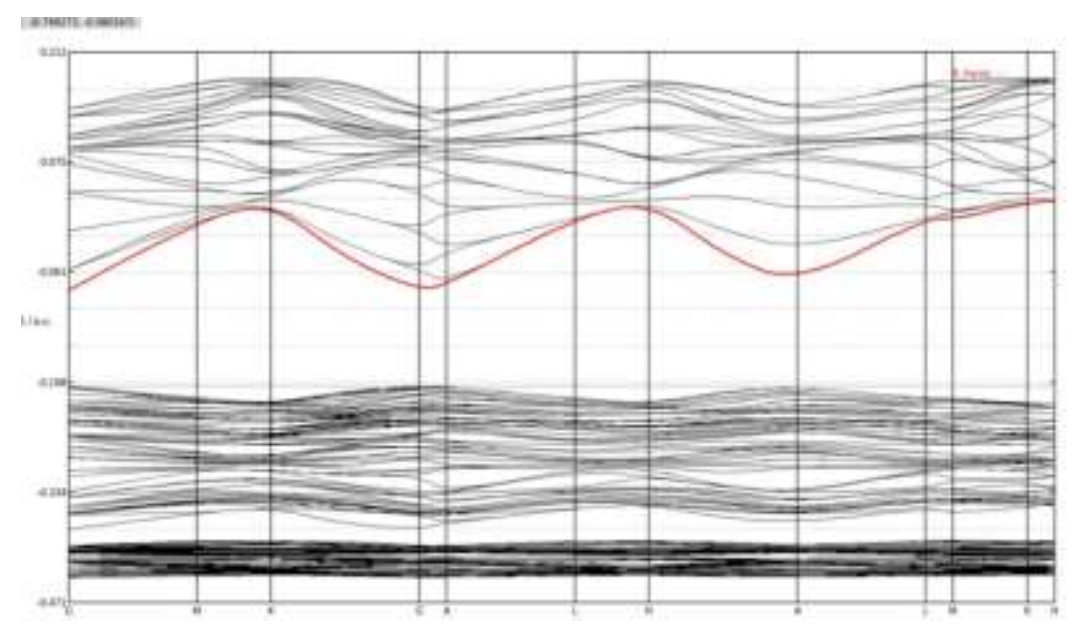

Figure2: Electronic Band Structure for $\mathrm{ZnO}$ (2x2x5supercell). Band Gap Between the Top of The Valence Band and The Bottom of The Conduction Band at Gamma Point $\approx 3.3 \mathrm{e}$ 


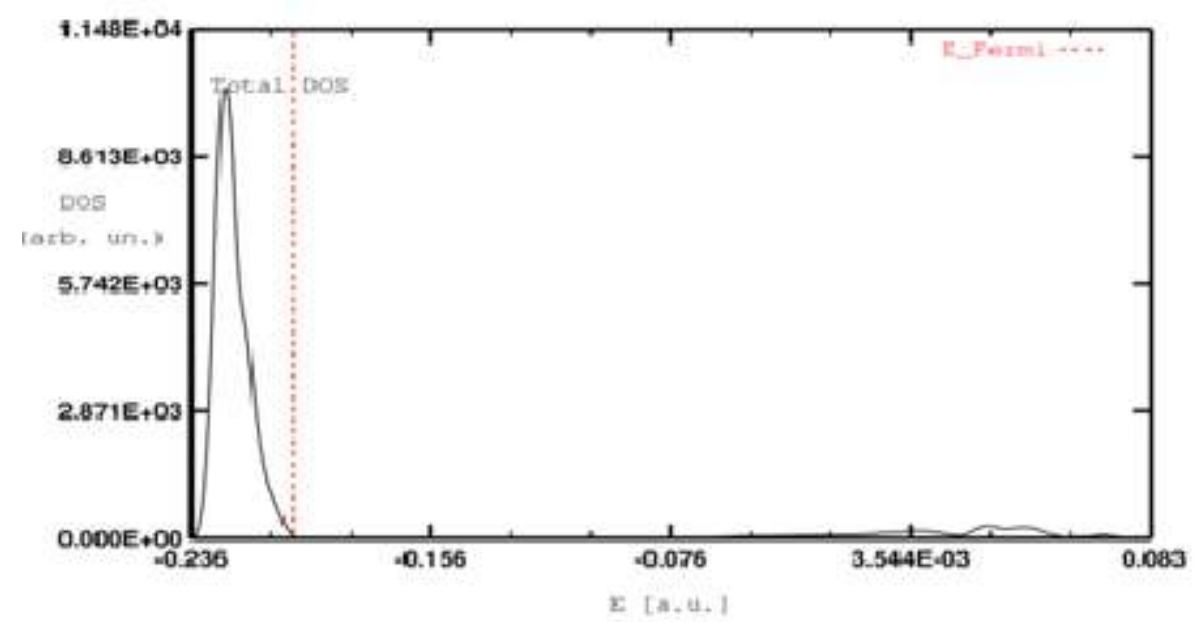

Figure3: Total Density of State $\mathrm{ZnO}$ crystal Energy measured from the Fermi Surface

\subsection{Zinc Oxide Surface Structures}

In this cases $2 \mathrm{D}$ system in crystal06 is used to calculate surface properties, slab option used to given thickness parallel to the given plane (100) of the 3D lattices, is defined by the number of layers, and number of atoms 40 per cell it is shown in figure4.

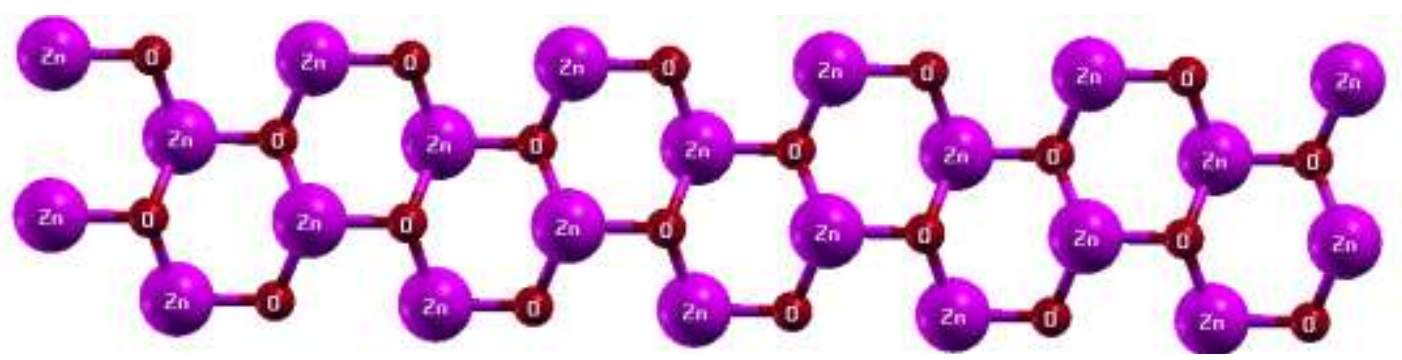

Figure4: Electronic Structures of $\mathrm{ZnO}$ Slab

\subsection{Band Structures of $\mathrm{ZnO}$ slab}

In Hexagonal system in plan $\left(\begin{array}{lll}1 & 0 & 0\end{array}\right)$, geometry for wave function dimensionality of the system using high symmetry point G-M, one obtained the value of the band-gap as shown in Figure5. From the result obtained the band-gap between upper band in valance band and lower band in conductive band $\approx 5.5 \mathrm{eV}$ at gamma point.

Figure6 shows the density of state. we can see that the band gap is wide than the bulk band gap $(3.3 \mathrm{eV})$ this increase in band gap mean this layer of material changed to isolated material. 


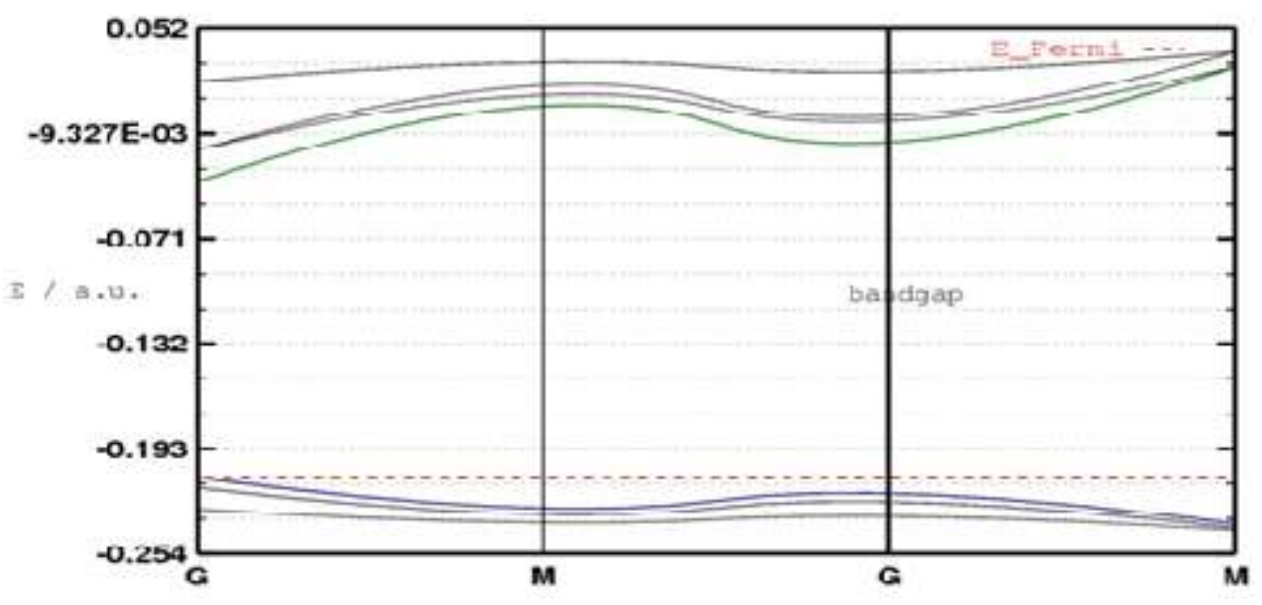

Figure5: Band Structures of $\mathrm{ZnO}$ Slab

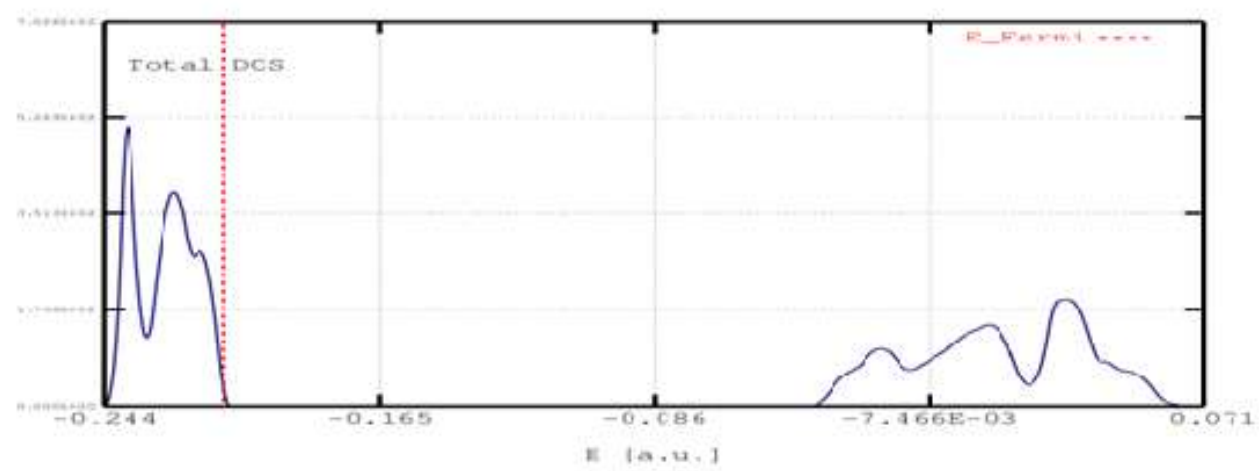

Figure6: Density of State Structures of ZnO Slab

\subsection{Substituting of $\mathrm{Li}$ in $\mathrm{ZnO}$ slab} Structure

Figure7 shows the substitution of one zinc atom by one Lithium atom on the surface of $\mathrm{ZnO}$ compound. This will enable us to study the effect of doping the Lithium atom on $\mathrm{ZnO}$ nanosheet.

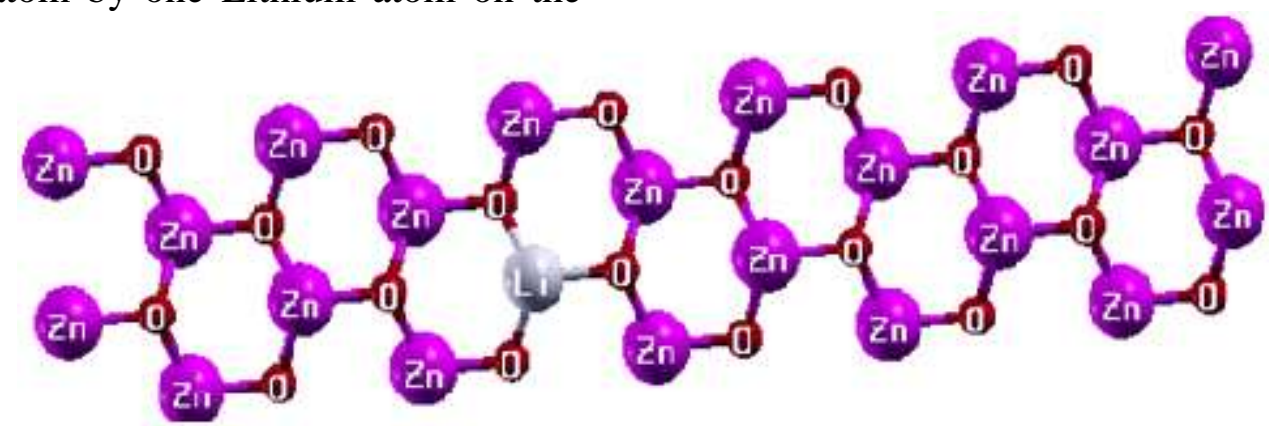

Figure7: Doped Li in $\mathrm{ZnO}$ Structure.

\subsubsection{Band structure of $\mathrm{Li}$ doped in ZnO Slab}

In Hexagonal system in plan ( $\left.\begin{array}{lll}1 & 0 & 0\end{array}\right)$, using high symmetry point G-M and obtained the band structure of $\mathrm{LiZnO}$, the value of the band-gap $\approx 4.66 \mathrm{eV}$. Figure8 shows that, decreases in band gap comparison to $\mathrm{ZnO}$ slab Band gap, this mean the effect of substitute lithium ion in $\mathrm{ZnO}$ slab decreases resistivity and consequently increasing in conductivity. But it is notes that the highest level of valance band is crossing the Fermi level and it is known that in case if one of the 
electron spin is crossing Fermi level the

material can be change to half-metal.

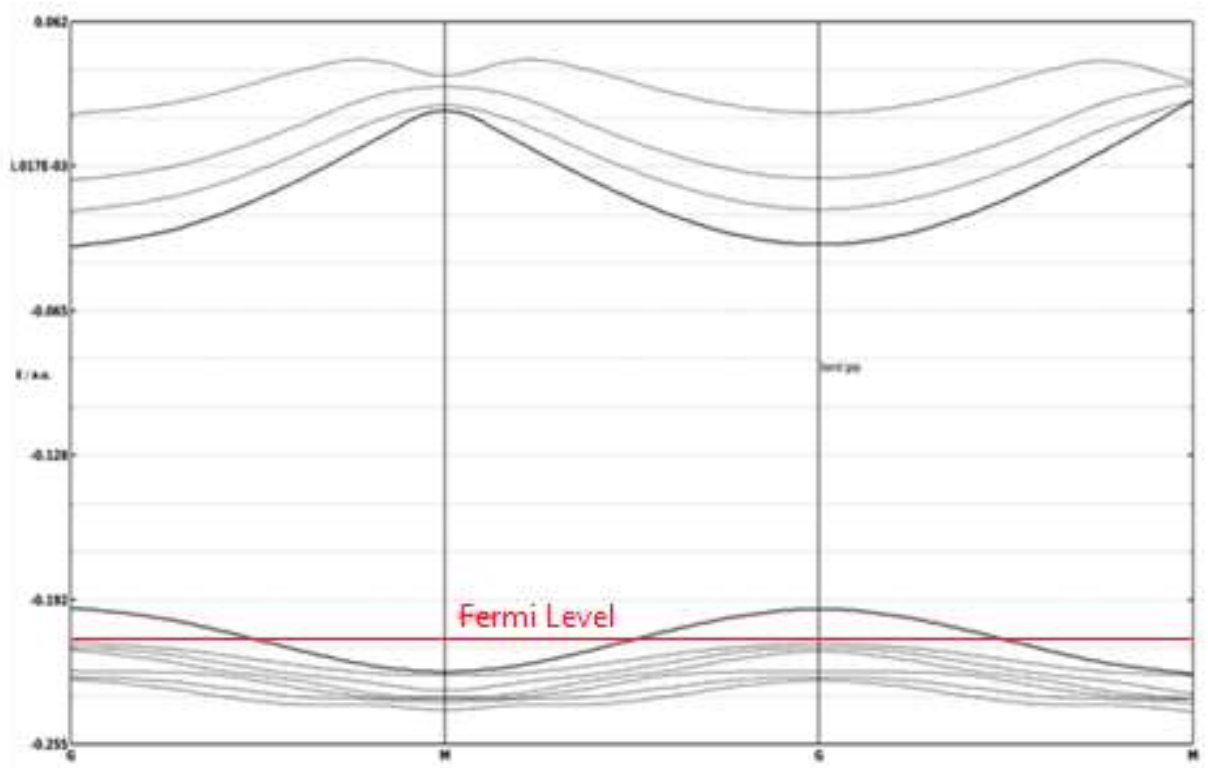

Figure8: Band Structures of Doped Li in ZnO SLAB

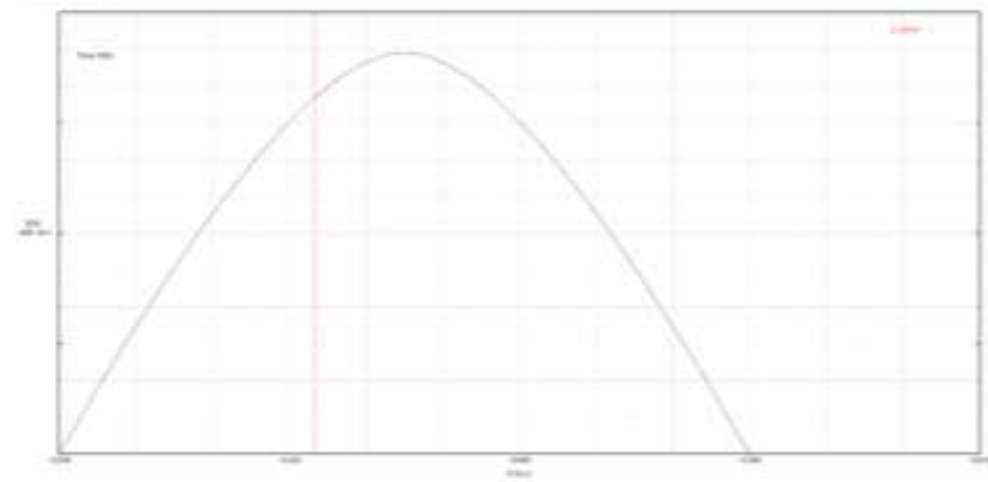

Figure 4.1: DOS Structure of Doped LI in ZnO SLAB

\section{Conclusion}

The resulting band gap of simulation of bulk zinc-oxide is $\approx 3.3 \mathrm{eV}$ which is in good agreement with the experimental band gap $\approx 3.4 \mathrm{eV}$. The band gap of the generated slab increases to $\approx 5.5$, mean isolated defects and exhibiting high resistivity.

Some reports indicate that $\mathrm{Li}$ substituting for $\mathrm{Zn}$ would be increases the resistivity, while the present results are different. The results show that there is reduction in band-gap and decreases in resistivity, predicting that Small amount of Lithium could change the zinc oxide from wide band semiconductor to half-metal compound.

\section{References}

Coleman, V.A., Jagadish, C., 2006. Chapter 1 - Basic Properties and Applications of $\mathrm{ZnO}$, in: Jagadish, Chennupati, Pearton, S. (Eds.), Zinc Oxide Bulk, Thin Films and Nanostructures. Elsevier Science Ltd, Oxford, pp. 1-20. https://doi.org/10.1016/B978008044722-3/50001-4 crystal17.pdf, n.d.

Kim, J.S., Lee, H.J., Seog, H.J., Kim, I.W., 2011. Dielectric and electrical properties of $\mathrm{Li}$-doped $\mathrm{ZnO}$ films. Journal of the Korean Physical Society $58,640-644$. 
Moezzi, A., McDonagh, A.M., Cortie, M.B., 2012. Zinc oxide particles: Synthesis, properties and applications. Chemical Engineering Journal 185-186, 1-22.

https://doi.org/10.1016/j.cej.2012.01.07 6

Phys. Rev. B 66, 073202 (2002) Origin of p-type doping difficulty in $\mathrm{ZnO}$ : The impurity perspective [WWW Document], n.d. URL https://journals.aps.org/prb/abstract/10.1 103/PhysRevB.66.073202 (accessed 1.19.20).

Phys. Rev. B 71, 155205 (2005) Theory of $\mathrm{Li}$ in $\mathrm{ZnO}$ : A limitation for Li-based \$p\$-type doping [WWW Document], n.d. URL https://journals.aps.org/prb/abstract/10.1
103/PhysRevB.71.155205 (accessed 1.19.20).

Physical Electronics and Nanotechnology - $\quad$ Linköping University [WWW Document], n.d. URL https://liu.se/en/research/physicalelectronics-and-nanotechnology (accessed 1.19.20).

Wall-E, AJa HGVd,2009 Fundamental of Zinc Oxide as Semiconductor. 2009. https://www.google.com/search?hl=ar\& $\mathrm{q}=$ Wall,+AJa+HGVd,+Fundamental+of+Zinc+ Oxide+as+Semiconductor.+2009. (accessed 1.19.20).

XCrySDen - (X-Window) Crystalline Structures and Densities [WWW Document], n.d. URL http://www.xcrysden.org./ (accessed 1.19.20). 